\title{
Dietary guidance during pregnancy and iodine nutrition: a qualitative approach
}

\author{
M. Bouga, M.E. Lean and E. Combet \\ Human Nutrition, School of Medicine, College of Medical Veterinary and Life Sciences, University of Glasgow, \\ Glasgow Royal Infirmary, Glasgow, UK
}

Iodine is important for the formation of thyroid hormones and infant neurodevelopment; however iodine intake, knowledge and awareness remain low amongst UK mothers ${ }^{(1-3)}$. Using qualitative study design, we aimed to explore mothers' perceptions of nutrition in pregnancy, focussing on points pertinent to iodine nutrition.

Women were recruited by snowball sampling using advertisements (in social media, relevant websites and places in the community) and word of mouth (May to November 2015). Inclusion criteria included UK residency, fluent English and being pregnant, having a child (younger than 2 years old) or trying to conceive. All participants were interviewed individually by phone or face-to-face using a topic guide. Interviews were transcribed verbatim and analysed with thematic analysis, using NVivo version 11 (QSR International).

Participants $(n=48)$ were pregnant $(38 \%)$, breastfeeding $(35 \%)$, planning to conceive $(10 \%)$ and $17 \%$ had a baby or toddler. Four main themes emerged from the interviews: $i$ ) dietary guidance received; $i$ ) iodine awareness, $i i i)$ acceptance of iodine rich products, difficulties and barriers towards consumption and $i v$ ) desirable characteristics of dietary guidance.

An emerging theme in women with multiple pregnancies $(n=11)$ was assumption of prior knowledge by health professionals, leading to less information being provided. This is an issue, considering that most participants were unaware of the importance of iodine, its sources and recommended intake, as previously shown ${ }^{(3)}$. Only women with nutrition/medical backgrounds were aware of the importance of iodine, its sources and recommended intake - information both surprised and concerned women once handed from the researcher. Written dietary advice was received by most during the first antenatal care appointment (around 12-14 weeks gestation), with an overt focus on foods to avoid and supplements ("I had very little directions from [the] doctor so I've been on the internet."). However, a clear theme of trust in the health services emerged, with midwives and general practitioners considered experts in pregnancy nutrition.

Attitude to dairy products was generally positive. Milk was recognised to alleviate heartburn symptoms and to be craved for pregnancy. In cases of dislike, women still tried to increase milk consumption via yogurts and/or cheese. The main barriers towards dairy consumption included taste (mainly for milk), (true or perceived) lactose intolerance or health conditions associated to dairy products (e.g. eczema), morning sickness and perceptions of unhealthiness associated with cheese products (fat, processed foods). It was perceived difficult to increase or maintain fish and/or seafood consumption, the main barrier to which was the general dislike of these foods, exacerbated by heartburn, morning sickness and change of taste and smell during pregnancy. Other stated reasons of fish exclusion were partner's and family preferences, lack of cooking skills, cost implications, low availability and habit of not buying/ eating it regularly. Even when there was a clear intention to consume fish and seafood, confusion over recommendations and worry of eating the wrong fish species were barriers to consumption.

Analysis highlighted a theme of clear commitment to change behaviour if prompted ("If I knew how important it was, I would increase it."). Key desirable features for nutrition information included clearer, more practical and easy to remember guidance, with focus on user-friendly documentation, focusing on portion size and foods rather than nutrients, with continued involvement of the health services as a trusted provider of that information.

In conclusion, the present study highlighted the importance of redirecting the way dietary guidance in pregnancy is provided. Future work should incorporate users' input to generate appropriate health promotion solutions.

1. Vanderpump MPJ, Lazarus JH, et al. Lancet. 2011;377(9782):2007-2012.

2. Bath SC, Sleeth ML, et al. Br J Nutr. 2014;112(10):1715-1723.

3. Combet E, Bouga M, et al. Br J Nutr. 2015;114(01):108-117. 